ARTíCULO

\title{
Variaciones estacionales de la comunidad de quitones (Mollusca: Polyplacophora) en una zona intermareal rocosa del sur del Golfo de México
}

Seasonal variations of the chiton community structure (Mollusca: Polyplacophora) in a rocky intertidal shore from the southern Gulf of Mexico

\section{Laura Sanvicente-Añorve ${ }^{*}$, Rodrigo Rodríguez-Vázquez ${ }^{2}$, Elia Lemus-Santana ${ }^{2}$, Miguel Alatorre-Mendieta ${ }^{1}$ y Martha Reguero ${ }^{1}$}

${ }^{1}$ Instituto de Ciencias del Mar y Limnología, Universidad Nacional Autónoma de México, Circuito Exterior S/N, Ciudad Universitaria, Delegación Coyoacán, 04510 Ciudad de México, México. *Autor para correspondencia: lesa@unam.mx ${ }^{2}$ Facultad de Ciencias, Universidad Nacional Autónoma de México, Circuito Exterior S/N, Ciudad Universitaria, Delegación Coyoacán, 04510 Ciudad de México, México

\begin{abstract}
The southern Gulf of Mexico is typically a coastal plain where rocky shores are scattered distributed. The goal of this study was to compare the chiton community structure of the rocky shore of Montepío during the dry and 'nortes' (stormy) weather periods, and to gather data on the relative growth of the dominant species. Sampling was carried out along a transect of $100 \times 2 \mathrm{~m}$ in the intertidal zone and 83 individuals were collected. Six species were identified: Lepidochitona rosea, Chaetopleura apiculata, Ischnochiton kaasi, Lepidochitona pseudoliozonis, Lepidochitona liozonis and Acanthochitona andersoni. Species richness $(S=4)$ and Shannon diversity $\left(1.36\right.$ and 1.29 bits ind $^{-1}$ in dry and stormy seasons) were very similar between the 2 seasons; however, only 2 species occurred in both periods, suggesting a strong seasonal exchange of species (50\%). This seasonal dynamics could be due to the discontinuity of the rocky substrate, which would not allow a rapid recovery of the populations after the impact of storms. Density of individuals was low $(<2.50$ ind. $10 \mathrm{~m}^{-2}$ ) due to 2 factors: the diurnal tide, which induces the individuals to $12 \mathrm{~h}$ of air exposure, and the low salinity, due to the proximity of two rivers. The relative growth of the dominant species (L. rosea and C. apiculata) was isometric for the length vs. width relationship and negatively allometric for the length vs. height relationship. In the southern Gulf of Mexico, sites such as Montepío may function as marine oases for obligate inhabitants of hard substrates.
\end{abstract}

Key words: Littoral rocky shore, community structure, species exchange, Montepío, biometric relationships

\begin{abstract}
Resumen.- La costa sur del Golfo de México es típicamente una llanura costera que alterna con salientes rocosas. Este estudio tuvo como objetivo comparar la estructura comunitaria de los quitones del litoral rocoso de Montepío, durante los períodos meteorológicos de secas y nortes y proporcionar datos sobre el crecimiento relativo de las especies dominantes. Se muestreó sobre un transecto de $100 \times 2 \mathrm{~m}$ en la zona intermareal y se recolectaron 83 individuos. Se identificaron 6 especies: Lepidochitona rosea, Chaetopleura apiculata, Ischnochiton kaasi, Lepidochitona pseudoliozonis, Lepidochitona liozonis y Acanthochitona andersoni. Las 2 épocas fueron muy similares en riqueza de especies $(S=4)$ y diversidad de Shannon (1,36 y 1,29 bits ind $^{-1}$ en secas y nortes); sin embargo, solo 2 especies ocurrieron en ambos períodos, lo que indica un fuerte recambio estacional de especies (50\%). Esta dinámica estacional se debería a la discontinuidad del sustrato rocoso, que no permitiría una recuperación rápida de las poblaciones después del impacto de los nortes. La densidad de organismos fue baja $\left(<2,50\right.$ ind. $\left.10 \mathrm{~m}^{-2}\right)$ debido a 2 factores: la marea diurna, que induce a los individuos a 12 $\mathrm{h}$ de exposición al aire, y la baja salinidad, debida a la cercanía a dos ríos. El crecimiento relativo de las especies dominantes (L. rosea y C. apiculata) fue isométrico para la relación largo vs. ancho y alométrico negativo para la relación largo vs. altura. En el sur del Golfo de México, sitios como Montepío podrían funcionar como oasis marinos para habitantes obligados de sustratos duros.
\end{abstract}

Palabras clave: Litoral rocoso, estructura comunitaria, recambio de especies, Montepío, relaciones biométricas

\section{INTRODUCCIÓN}

Los moluscos poliplacóforos o quitones son animales exclusivamente marinos que se distribuyen en todos los océanos del mundo, desde el ecuador a los polos (Kaas
\& Van Belle 1985a, García-Ríos 2003), desde la zona intermareal hasta la hadal (Schwabe 2008) y en sitios con fuerte energía de oleaje o zonas muy protegidas (Sigwart et al. 2015). Estos organismos generalmente habitan 
sustratos duros y pueden encontrarse en costas rocosas y zonas coralinas, o bien, asociados a conchas de otros moluscos o entre la vegetación (Lyons \& Moretzsohn 2009).

En la zona intermareal rocosa, los quitones y el resto de la fauna han sobrevivido al constante embate de las olas y a la desecación, los factores físicos más estresantes en ese tipo de ambientes (Bustamante et al. 1997). En esta zona, la estabilidad del sustrato contrasta con la de las comunidades que lo habitan. Little \& Kitching (1996) mencionan que los cambios intra o interanuales en la fauna dependen en gran medida del grado de protección de la zona, la proporción de cobertura vegetal, las fuertes tormentas y oleaje asociado, así como la depredación, factores todos cuya interacción induce a cambios en la dominancia de las especies.

Dentro de la trama trófica de las costas rocosas, los quitones desempeñan un papel muy importante en la transferencia de energía desde los productores primarios a los consumidores. Estos animales se alimentan principalmente de algas adheridas al sustrato (Kaas \& Van Belle 1985a) y, para evitar la depredación, son capaces de adquirir la coloración de su hábitat o refugiarse en las oquedades. No obstante, las aves marinas, peces, crustáceos u otros moluscos pueden acceder a ellos para alimentarse. Otros factores de mortalidad natural son períodos largos de lluvias o de marea excepcionalmente baja (García-Ríos 2003).

En comparación con otros invertebrados marinos, los quitones exhiben poca variabilidad morfológica (Sigwart et al. 2015). Su estructura general, consistente de cuerpo oval aplanado con 8 valvas articuladas y un gran pie muscular adherido al sustrato, ha tenido un gran valor adaptativo en los diferentes ambientes y se ha mantenido por más de 500 millones de años (Eernisse 2007). La proporción entre el largo y el ancho del cuerpo de un quitón define diferentes formas: oval, oval-amplia, ovalelongada o vermiforme, según las diferentes especies (Schwabe 2010). La forma y relación área-volumen de un organismo son fundamentales para el intercambio de fluidos y contrarrestar problemas de desecación (Lowell 1984). A pesar del valor adaptativo de su forma, las relaciones biométricas y crecimiento relativo de los quitones han sido poco estudiados (Baxter 1982, Baxter \& Jones 1986, Watters 1991).

La costa sur del Golfo de México es típicamente una llanura costera de amplitud variable que alterna con salientes rocosas de origen volcánico e importantes cuerpos de agua de origen continental (Moreno-Casasola 2004). Un importante fenómeno meteorológico afecta esta región: los nortes. Éstos son masas de aire frío provenientes del norte que irrumpen en el sur del golfo con velocidades de hasta $110 \mathrm{~km} \mathrm{~h}^{-1}$, producen descensos de temperatura de hasta $15^{\circ} \mathrm{C}$ en un día e inducen una alta precipitación (Acevedo-Rosas \& Díaz-Peón 2006); no obstante, existen también períodos de seca o baja precipitación pluvial. En el Golfo de México, las investigaciones dirigidas a los poliplacóforos son escasas. Lyons \& Moretzsohn (2009) proporcionaron una lista actualizada de las especies, Reyes-Gómez et al. (2017) refirieron la fauna de quitones del Arrecife Alacranes, en tanto que Kaas (1993) y García-Ríos (2015) describieron sendas nuevas especies. El objetivo de este estudio fue analizar la estructura comunitaria de los quitones en una zona intermareal rocosa del sur del Golfo de México en 2 épocas contrastantes del año: secas (abril) y nortes (octubre) y proporcionar información sobre el crecimiento relativo de las especies dominantes en la comunidad. Se hipotetiza un cambio importante en el microhábitat y/o composición de las especies en respuesta a diferencias ambientales.

\section{Materiales y MÉtodos}

El sitio de recolección se ubica en las coordenadas geográficas $18^{\circ} 38,75^{\prime} \mathrm{N}$ y $95^{\circ} 5,66^{\prime} \mathrm{W}$, en la localidad de Montepío, Veracruz (sur del Golfo de México), localizada aproximadamente a $500 \mathrm{~m}$ al noreste de la desembocadura de los ríos Col y Máquinas (Fig. 1). Situada en una zona volcánica, la morfología costera de Montepío está influida por la acción del oleaje sobre la roca basáltica que produce acantilados verticales y abruptas entrantes, así como por el depósito de sedimentos en las desembocaduras de ríos que forman barras y playas (Martín-del Pozzo 1997). En relación al régimen de vientos y lluvias, la región se caracteriza por 3 períodos meteorológicos: 'nortes' de octubre a febrero, caracterizado por fríos vientos del norte y fuertes tormentas ocasionales, 'secas' de febrero a mayo, tipificado por vientos del este y del sureste y baja precipitación, y 'lluvias' de junio a septiembre, con el mismo patrón de vientos pero alta precipitación (YáñezArancibia \& Day 1982). Las mareas son de tipo diurno con una amplitud media de 0,26 m (SMN 2009) ${ }^{1}$.

\footnotetext{
${ }^{1}$ SMN. 2009. Servicio Mareográfico Nacional. Universidad Nacional Autónoma de México. Instituto de Geofísica. <http://www.mareografico.unam.mx>
} 


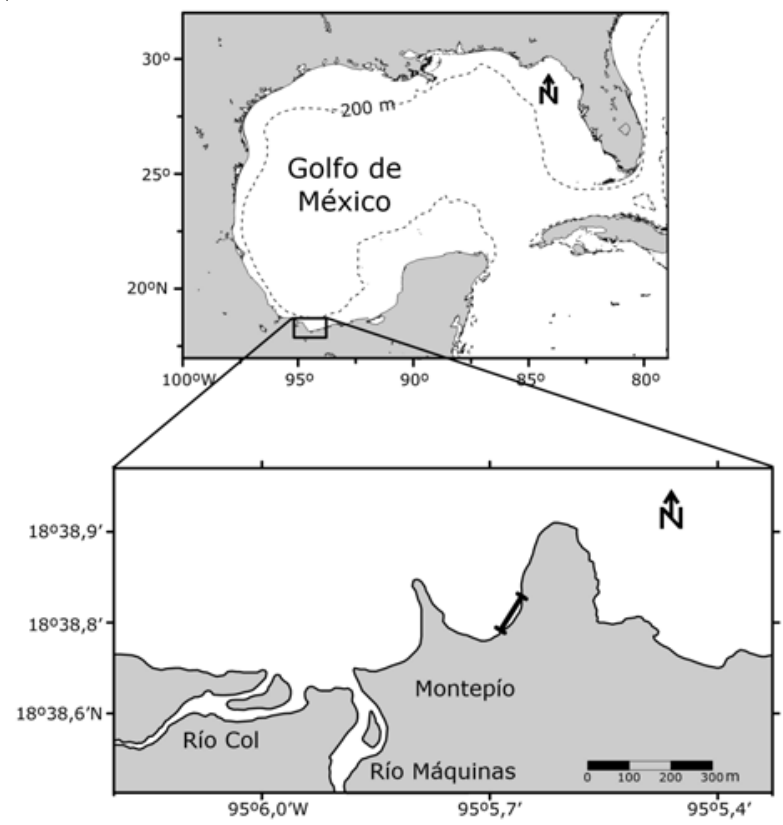

El estudio se llevó a cabo en abril (época de secas) y octubre (época de nortes) de 2014. El muestreo se efectuó en la zona intermareal sobre un transecto de $100 \mathrm{~m}$ de longitud y $2 \mathrm{~m}$ de ancho, durante la marea baja y utilizando técnicas de buceo libre y extracción manual con una espátula. Para evitar la contracción de los organismos recolectados, éstos se sumergieron en una solución de aceite de clavo diluida con agua de mar durante $24 \mathrm{~h}$. Al término de este proceso, los ejemplares se fijaron con etanol al $70 \%$. Adicionalmente, se midieron la temperatura y la salinidad del agua con ayuda de un termosalinómetro YSI, con precisiones de $\pm 0,01^{\circ} \mathrm{C}$ en temperatura $\mathrm{y} \pm 0,01$ en salinidad.

En el laboratorio, los quitones se identificaron al nivel taxonómico de especie siguiendo los criterios de literatura especializada (Kaas \& Van Belle 1985b, 1987, 1990; Ferreira 1987, Lyons 1988, García-Ríos 2003, 2015; García-Ríos \& Álvarez-Ruiz 2011). La densidad de las especies se estandarizó a $10 \mathrm{~m}^{2}$ de área (ind. $10 \mathrm{~m}^{-2}$ ). Para conocer y comparar la diversidad entre épocas (secas y nortes), se estimó la riqueza de especies $(S)$ y la diversidad de Shannon $\left(H^{\prime}\right)$. Además, se examinó el crecimiento relativo de las especies dominantes $(n \geq 23)$. Para ello, de cada espécimen se midió el largo $(L)$, el ancho $(A)$ y la altura $(H)$, con ayuda de un micrómetro incorporado a un microscopio estereoscópico Zeiss ${ }^{\circledR}$ modelo Stemi. Para analizar el grado de alometría en el crecimiento del organismo, cada par de variables se ajustó a una ecuación de tipo potencial $\left(Y=b X^{k}\right)$, en donde $X$ representa la
Figura 1. Localización geográfica del sitio de estudio: Montepío, Veracruz, sur del Golfo de México / Geographical location of the study area: Montepío, Veracruz, southern Gulf of Mexico

variable independiente $(L), Y$ es la variable dependiente ( $A$ o $H$ ) y $k$ es el parámetro de crecimiento o pendiente en la regresión lineal de la ecuación potencial linealizada (transformada a logaritmos). El valor de $k$ indica si el crecimiento en los organismos es de tipo isométrico $(k=$ 1), alométrico positivo $(k>1)$ o alométrico negativo $(k<$ $1)$. Un crecimiento isométrico indica que ambas variables crecen en la misma proporción, en tanto que un crecimiento de tipo alométrico indica que una de ellas crece más rápidamente. Para detectar si el valor de $k$ difiere o no significativamente de 1 , se usó la prueba:

$$
t=\frac{|k-1|}{S_{k}} \sim T(n-2)
$$

en donde, $S_{k}=\frac{S_{y \cdot x}}{S_{x} \sqrt{n-1}}$ y $S_{y \cdot x}=S_{y} \sqrt{\frac{\left(1-r^{2}\right)(n-1)}{(n-2)}}$

con $S_{*}$ igual a la desviación estándar de $X$ o $Y, r^{2}$ el coeficiente de determinación y $n$ el número de datos (Zar 1999). Estas pruebas se efectuaron con un nivel de significancia de $\alpha=0,05$. 


\section{Resultados}

Ambientalmente, la zona de estudio estuvo caracterizada por temperaturas altas $\left(29,45\right.$ a $\left.29,9^{\circ} \mathrm{C}\right)$ y salinidades bajas (25,25 a 27), sin diferencias contrastantes entre los meses de exploración. La influencia de los ríos Col y Máquinas se evidenció en los valores bajos de salinidad. En términos relativos, la energía del oleaje fue mayor en octubre que en abril.

Considerando ambas épocas de muestreo, se recolectó un total de 83 individuos y se identificaron 6 especies (Fig. 2): Lepidochitona rosea Kaas, 1972, Chaetopleura apiculata (Say, 1834), Ischnochiton kaasi Ferreira, 1987, Lepidochitona liozonis (Dall \& Simpson, 1901), Lepidochitona pseudoliozonis García-Ríos, 2015 y Acanthochitona andersoni Watters, 1981, entre las que destacó la alta abundancia de las 2 primeras (Tabla 1). Las primeras 5 especies pertenecen a la familia Ischnochitonidae, en tanto que A. andersoni es miembro de la familia Acanthochitonidae. Todos los especímenes recolectados fueron de talla pequeña $(<1,4 \mathrm{~cm})$.
La densidad de quitones fue baja en general: 1,65 ind. $10 \mathrm{~m}^{-2}$ en nortes y 2,50 ind. $10 \mathrm{~m}^{-2}$ en secas. La riqueza de especies $(S)$ en ambas épocas fue la misma $(S=4)$ y la diversidad de Shannon ( $H^{\prime}$ ') mostró valores semejantes, secas con 1,36 bits ind ${ }^{-1}$ y nortes con 1,29 bits ind ${ }^{-1}$. Si bien los valores de diversidad y riqueza de especies fueron similares, hubo diferencias notables en la composición de especies entre las 2 épocas (Tabla 1). De hecho, solo C. apiculata y L. pseudoliozonis se encontraron en ambas épocas, lo que significó un recambio de 50\% de las especies. La especie dominante en abril fue L. rosea, mientras que en octubre fue $C$. apiculata.

Durante la época de secas, los individuos se encontraron generalmente sobre el sustrato, en tanto que durante la época de nortes, los quitones estaban más al abrigo de las inclemencias ambientales. Lepidochitona pseudoliozonis y L. liozonis se encontraron sobre tapetes algales; esta última especie, sobre colonias de algas incrustantes de tonos marrón-rojizas. Lepidochitona rosea se encontró sobre las rocas, en ocasiones formando
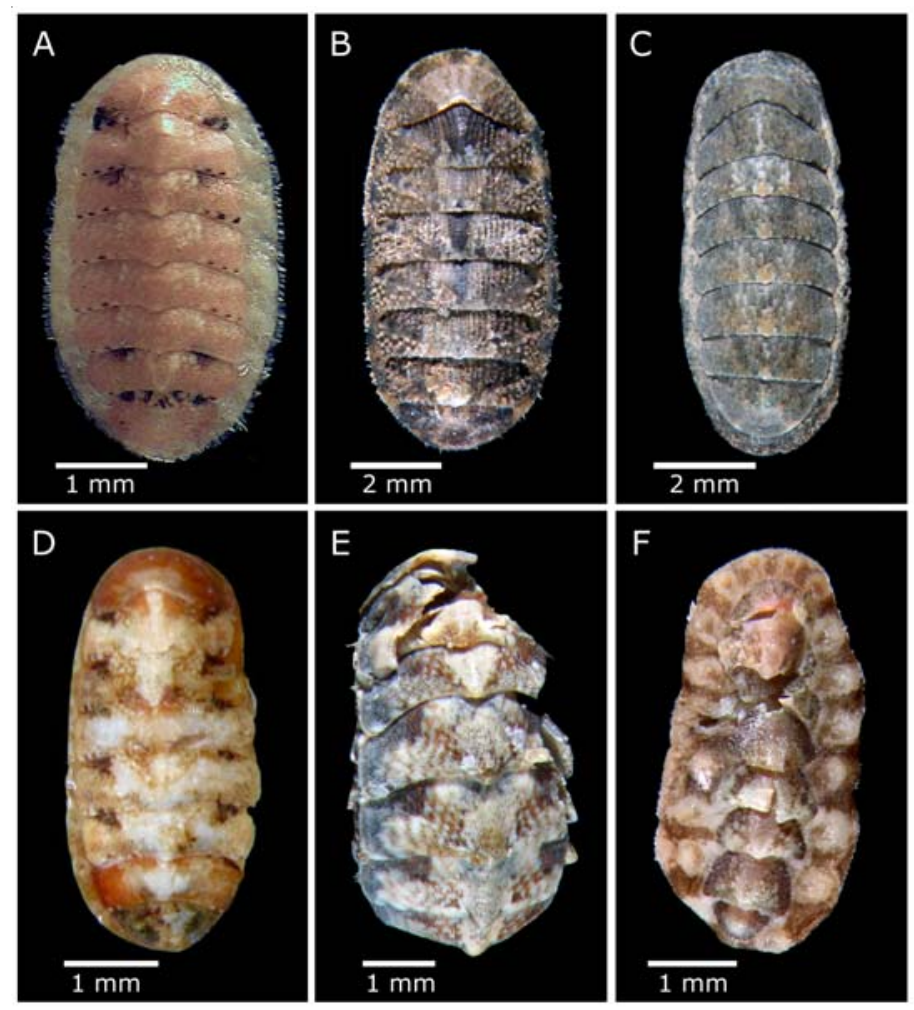

Figura 2. Especies de poliplacóforos recolectadas en Montepío, Veracruz, sur del Golfo de México. A. Lepidochitona rosea, B. Chaetopleura apiculata, C. Ischnochiton kaasi, D. Lepidochitona liozonis, E. Lepidochitona pseudoliozonis, F. Acanthochitona andersoni / Polyplacophoran species collected in Montepío, Veracruz, southern Gulf of Mexico 
Tabla 1. Número de individuos por especie $(n)$ y densidad (ind. $10 \mathrm{~m}^{-2}$ ) de los quitones recolectados en 2 épocas del año en la zona intermareal rocosa de Montepío, Veracruz, sur del Golfo de México / Number of individuals per species $(n)$ and density (ind. $10 \mathrm{~m}^{-2}$ ) of chitons collected during 2 seasons in the intertidal rocky shore of Montepío, Veracruz, southern Gulf of Mexico

\begin{tabular}{lcccccccc}
\hline & \multicolumn{2}{c}{ Secas (abril) } & & \multicolumn{2}{c}{ Nortes (octubre) } & & \multicolumn{2}{c}{ Ambas épocas } \\
\cline { 2 - 3 } & $n$ & ind. $10 \mathrm{~m}^{-2}$ & & $n$ & ind. $10 \mathrm{~m}^{-2}$ & & $n$ & ind. $10 \mathrm{~m}^{-2}$ \\
\hline Acanthochitona andersoni & - & - & & 1 & 0,05 & & 1 & 0,03 \\
Chaetopleura apiculata & 4 & 0,20 & & 19 & 0,95 & & 23 & 0,58 \\
Ischnochiton kaasi & - & - & & 12 & 0,60 & & 12 & 0,30 \\
Lepidochitona liozonis & 3 & 0,15 & & - & - & & 3 & 0,08 \\
Lepidochitona pseudoliozonis & 9 & 0,45 & & 1 & 0,05 & & 10 & 0,25 \\
Lepidochitona rosea & 34 & 1,70 & & - & - & & 34 & 0,85 \\
Total & 50 & 2,50 & & 33 & 1,65 & & 83 & 2,08 \\
\hline
\end{tabular}

conglomerados de hasta 7 individuos. Ischnochiton kaasi y A. andersoni, presentes solo durante nortes, fueron encontradas debajo de rocas sueltas.

Se examinó el crecimiento relativo de los individuos de las 2 especies dominantes: $L$. rosea $(n=34)$ y C. apiculata ( $n=23)$. El intervalo de tallas para $L$. rosea fue de 3,8 a 7,1 mm de largo, y para C. apiculata, de 3,2 a 13,1 mm. El parámetro $k$ de la relación largo vs. ancho en ambas especies no mostró ser significativamente diferente del valor de 1 ( $L$. rosea: $t=1,28$, g.l. $=32, P>0,05$; C. apiculata: $t=0,37$, g.l. $=21, P>0,05$ ), por lo que el crecimiento en ese par de variables es isométrico. Sin embargo, el parámetro de crecimiento $k$ de la relación largo vs. altura, fue significativamente menor a 1 ( $L$. rosea: $t=5,98$, g.l. $=32, P$ $<0,05$; C. apiculata: $t=4,30$, g.l. $=21, P<0,05$ ), lo que indica un crecimiento de tipo alométrico negativo en ambas especies (Tabla 2), es decir, la altura disminuye en proporción al largo conforme el animal crece.

\section{Discusión}

La composición tuvo un recambio de 50\% de una época a otra: L. liozonis y $L$. rosea solo se encontraron en secas, I. kaasi y A. andersoni solo en nortes, en tanto que $L$. pseudoliozonis y C. apiculata en ambas épocas. Refiriendo a los crustáceos de la zona intermareal de Montepío, Hernández-Álvarez \& Álvarez (2007) quienes muestrearon mensualmente observaron el mayor recambio de especies (73\%) de febrero a marzo, y también, una alta ocurrencia de especies raras (47\%) durante el ciclo anual. Los autores arguyen que la incidencia de los nortes o fuertes tormentas tropicales impide el establecimiento de especies por lapsos largos. Además, la discontinuidad del hábitat rocoso en el litoral del sur del golfo podría ser otra causa del alto recambio de especies: la ausencia de un gran stock poblacional debido a la discontinuidad del
Tabla 2. Ajuste a una ecuación de tipo potencial y tipo de crecimiento de las especies dominantes de quitones recolectados en la zona intermareal rocosa de Montepío, Veracruz, sur del Golfo de México / Fit to a power equation and type of growth of the dominant chiton species collected in the intertidal rocky shore of Montepío, Veracruz, southern Gulf of Mexico

\begin{tabular}{|c|c|c|c|}
\hline Especie & $n$ & Ecuación & Crecimiento \\
\hline \multicolumn{4}{|l|}{ Largo vs. Ancho } \\
\hline Chaetopleura apiculata & 23 & $\mathrm{Y}=0,5672 \mathrm{X}^{0,9877}$ & Isométrico \\
\hline Lepidochitona rosea & 34 & $Y=0,4846 X^{1,0864}$ & Isométrico \\
\hline \multicolumn{4}{|l|}{ Largo vs. Altura } \\
\hline Chaetopleura apiculata & 23 & $Y=0,3313 X^{0,8169}$ & Alométrico negativo \\
\hline Lepidochitona rosea & 34 & $\mathrm{Y}=0,4723 \mathrm{X}^{0,6161}$ & Alométrico negativo \\
\hline
\end{tabular}

hábitat dificulta el restablecimiento de las poblaciones después de un disturbio natural (Hernández-Álvarez \& Álvarez 2007). Para el Golfo de México, los modelos de cambio climático predicen un aumento del nivel del mar entre 8 y $50 \mathrm{~cm}$ en los próximos 100 años, dependiendo de la topografía costera y su vulnerabilidad a las fuertes tormentas (Twilley et al. 2001). Un aumento en el nivel del mar, como apuntan las predicciones, cubriría algunas puntas rocosas o desplazaría verticalmente el hábitat de las especies que ocupan los sustratos duros.

Durante nortes la energía del oleaje fue mayor que durante secas, como resultado de diferencias en la velocidad del viento: $5,9 \mathrm{~m} \mathrm{~s}^{-1}$ en octubre y $4,4 \mathrm{~m} \mathrm{~s}^{-1}$ en abril (CNA 1999). En consecuencia, los individuos se encontraron en sitios más protegidos durante nortes y en zonas más expuestas durante la época de secas. De acuerdo con Smith (2013), las oquedades y espacios bajo las rocas generan microhábitats de protección contra la energía del oleaje, desecación y ataques de depredadores. Tal podría ser el caso de las 4 especies registradas en nortes, encontradas debajo de rocas sueltas. En secas, 
las algas tuvieron un papel preponderante como microhábitat de los quitones, particularmente para $L$. pseudoliozonis y L. liozonis. Esta última especie se encontró sobre colonias de algas incrustantes color marrón-rojizo, microhábitat donde puede mimetizarse con su entorno (García-Ríos 2015) y pasar inadvertida a sus depredadores. Lepidochitona rosea se encontró sobre las rocas, en grupos de hasta 7 individuos. Además de las ventajas que ofrece la agregación para evitar la desecación (Smith 2013), la conducta gregaria de los individuos es una táctica para mermar la depredación (Coleman et al. 1999).

La dominancia en la comunidad cambió estacionalmente: $L$. rosea fue la especie dominante en secas (abril) y $C$. apiculata en nortes (octubre). Lepidochitona rosea se distribuye desde el sur del Golfo de México y Mar Caribe hasta Brasil (García-Ríos 2003, Hernández-Pérez 2015) y se encuentra preferentemente sobre algas, hasta $2 \mathrm{~m}$ de profundidad (García-Ríos 2003). Chaetopleura apiculata se distribuye desde Massachusetts hasta el Caribe, incluyendo el Golfo de México (Kaas \& Van Belle 1987, Reyes-Gómez \& Salcedo-Vargas 2002, Lyons \& Moretzsohn 2009), sobre las rocas o conchas de otros moluscos (Lyons \& Moretzsohn 2009). A $32 \mathrm{~km}$ al sureste de Montepío, en el arrecife coralino La Perla del Golfo, estas 2 especies fueron designadas como 'común’ la primera y ‘dominante’ la segunda (Hernández-Pérez 2015), pero están ausentes del Arrecife Alacranes (Reyes-Gómez et al. 2017), situado a $695 \mathrm{~km}$ al noreste de Montepío, en el Banco de Campeche. Es probable que el corto periodo larvario (menor a una semana) de los quitones sea la causa principal de su baja dispersión (García-Ríos 2003).

La densidad de quitones registrada en Montepío fue baja: 1,65 ind. $10 \mathrm{~m}^{-2}$ en nortes y 2,50 ind. $10 \mathrm{~m}^{-2}$ en secas. Estos valores son al menos un orden de magnitud menor a los observados para la costa de California (Connor 1975), el litoral del Pacífico mexicano (Galeana-Rebolledo et al. 2014) y la región central de Chile (Otaíza \& Santelices 1985). Dos causas principales pueden influir en la baja densidad de quitones en Montepío: la cercanía a la desembocadura de 2 sistemas fluviales y el tipo de marea. La proximidad de los ríos Col y Máquinas induce a un descenso en la salinidad de la zona costera. Al ser el hábitat de los quitones esencialmente marino, es probable que un descenso en la salinidad aminore las poblaciones y solo algunas especies sean capaces de tolerar tales condiciones. Por ejemplo, en varios sitios de la costa de Nueva Zelanda, Boyle (1970) registró 10 especies, de las cuales solo Sypharochiton pellisekpentis (Quoy \&
Gaimard, 1835) fue capaz de tolerar un amplio intervalo de salinidad (13 a 45). Por otro lado, se ha documentado que el sitio de estudio es una de las pocas localidades en el mundo cuya marea es de tipo diurno (USNOO 1965), es decir, con un solo período de marea baja durante el día. En términos ecológicos, esto significa que los organismos de la zona intermareal están 12 horas expuestos al aire, sujetos a la desecación y cambios drásticos de temperatura. Algunos mecanismos para contrarrestar el estrés provocado por estos factores son: el uso de microhábitats sombreados con una humedad mayor que el resto de la roca circundante y el comportamiento gregario de los individuos (Smith 2013). Por ejemplo, la especie dominante durante la época de secas, $L$. rosea, se encontró en pequeños grupos, táctica que evita la desecación y depredación (Coleman et al. 1999, Smith 2013).

El crecimiento relativo de los individuos de las especies dominantes, L. rosea y C. apiculata, fue similar: isométrico para la relación largo vs. ancho y alométrico negativo para la relación largo vs. altura (Tabla 2). Watters (1991) analizó el crecimiento relativo de varias especies de quitones y mencionó que generalmente los miembros de la familia Ischnochitonidae presentan un crecimiento isométrico en la relación largo vs. ancho. No existen antecedentes sobre el crecimiento relativo de estas especies, pero para la población de Lepidochitona cinerea (Linnaeus, 1767) de las islas Orcadas (Escocia), Baxter (1982) encontró un crecimiento de tipo isométrico para ambas relaciones. Para la población de Tonicella marmorea (Fabricius, 1780) de la costa oeste de Escocia, Baxter \& Jones (1986) notaron -en la relación largo vs. ancho- un crecimiento de tipo isométrico para los individuos de algunos sitios de muestreo y alométrico negativo para otros. Estos resultados enaltecen la importancia del estudio de las relaciones biométricas en el enriquecimiento de los criterios taxonómicos que definen a las especies, así como en la identificación de variaciones morfológicas intraespecíficas.

De este estudio, se concluye que los cambios meteorológicos sobre el litoral rocoso del sur del Golfo de México tienen una gran influencia sobre la estructura de la comunidad de quitones. La alta tasa de recambio de las especies entre secas y nortes confirma el carácter dinámico de la comunidad de invertebrados, debido principalmente al impacto de los nortes y a la discontinuidad del hábitat rocoso. La baja densidad poblacional obedece a 2 factores principales: la baja salinidad derivada de la influencia de los ríos cercanos y 
la marea de tipo diurno, que implica que los organismos estén expuestos al aire por $12 \mathrm{~h}$. Los datos proporcionados sobre el crecimiento relativo de los individuos de las 2 especies dominantes amplían el conocimiento sobre las relaciones biométricas de este interesante grupo. A pesar del fuerte estrés físico al que está sujeto Montepío (la marea diurna, la baja salinidad y el impacto de los nortes), este sitio podría funcionar como un oasis marino para especies que necesitan de un sustrato duro para establecerse.

\section{Agradecimientos}

Los autores agradecen a G. Aguilar-Estrada, J. HernándezPérez y K. Rubio-Sandoval por su ayuda en los trabajos de campo y a M.B. Mendoza-Garfias, A.I. Bieler-Antolín y F. Zavala-García por su valiosa colaboración en las etapas posteriores de este estudio. Un agradecimiento especial a los revisores por la concienzuda crítica a este trabajo.

\section{LITERATURA CITADA}

Acevedo-Rosas F \& AL Díaz-Peón. 2006. Principales fenómenos meteorológicos que afectaron el estado de Veracruz en 2005. Centro de Previsión del Golfo de México, Comisión Nacional del Agua, Veracruz. <http:// www.iingen.unam.mx/es-mx/Publicaciones/Libros/ LibroInundaciones2005/04.pdf>

Baxter JM. 1982. Allometric and morphological variations of whole animal and valve dimensions in the chiton Lepidochitona cinereus (L.) (Mollusca: Polyplacophora). Journal of Molluscan Studies 48: 275-282.

Baxter JM \& AM Jones. 1986. Allometric and morphological characteristics of Tonicella marmorea (Fabricius, 1780) populations (Mollusca: Polyplacophora: Ischnochitonidae). Zoological Journal of the Linnean Society 88: 167-177.

Boyle PR. 1970. Aspects of the ecology of a littoral chiton, Sypharochiton pellisekpentis (Mollusca: Polyplacophora), New Zealand. Journal of Marine and Freshwater Research 4: 364-384.

Bustamante RH, GM Branch \& S Eekhout. 1997. The influences of physical factors on the distribution and zonation patterns of South African rocky-shore communities. South African Journal of Marine Science 18: 119-136.

CNA. 1999. Datos climáticos en Veracruz, México 1917-1998, 29 pp. Comisión Nacional del Agua, Gerencia Estatal en Veracruz, Centro de Previsión del Golfo de México, México.

Coleman RA, JD Goss-Custard, SEA Durell \& SJ Hawkins. 1999. Limpet Patella spp. consumption by oystercatchers Haematopus ostralegus: a preference for solitary prey items. Marine Ecology Progress Series 183: 253-261.
Connor MS. 1975. Niche apportionment among the chitons Cyanoplax hartwegii and Mopalia muscosa and the limpets Collisella limatula and Collisella pelta under the brown alga Pelvetia fastigiata. The Veliger 18(suppl.): 9-17.

Eernisse DJ. 2007. Chitons. In: Denny MW \& SD Gaines (eds). Encyclopedia of tidepools and rocky shores, pp. 127-133. University of California Press, Berkeley.

Ferreira JA. 1987. Two new species of Ischnochiton (Mollusca: Polyplacophora) in the western central Atlantic. Bulletin of Marine Science 40: 145-151.

Galeana-Rebolledo L, R Flores-Garza, A Reyes-Gómez, S García-Ibáñez, P Flores-Rodríguez, C TorreblancaRamírez \& A Valdés-González. 2014. Species richness and community structure of class Polyplacophora at the intertidal rocky shore on the marine priority region no. 33, Mexico. Open Journal of Ecology 4: 43-52.

García-Ríos CI. 2003. Los quitones de Puerto Rico, 208 pp. Isla Negra Editores, San Juan.

García-Ríos CI. 2015. Lepidochitona pseudoliozonis, una nueva especie de quitón (Polyplacophora: Ischnochitonidae) del norte del Caribe. Revista de Biología Tropical 63: 369-384.

García-Ríos CI \& M Álvarez-Ruiz. 2011. Diversidad y microestructura de quitones (Mollusca: Polyplacophora) del Caribe de Costa Rica. Revista de Biología Tropical 59: 129-136.

Hernández-Álvarez C \& F Álvarez. 2007. Changes in the crustacean community of a tropical rocky intertidal shore: is there a pattern? Hidrobiológica 17: 25-34.

Hernández-Pérez JR. 2015. Estudio faunístico de los moluscos del arrecife 'La Perla del Golfo', Veracruz, México. Tesis de Licenciatura, Facultad de Ciencias, Universidad Nacional Autónoma de México, 76 pp.

Kaas P. 1993. Ischnochiton mexicanus, a new abyssal chiton from the Gulf of Mexico (Polyplacophora, Ischnochitonidae). Basteria 57: 107-109.

Kaas P \& RA Van Belle. 1985a. Monograph of living chitons (Mollusca: Polyplacophora). Vol. 1. Order Neoloricata: Lepidopleurina, 240 pp. Brill-Backhuys, Leiden.

Kaas P \& RA Van Belle. 1985b. Monograph of living chitons (Mollusca: Polyplacophora). Vol. 2. Suborder Ischnochitonina. Ischnochitonidae. Schizoplacinae, Callochitoninae and Lepidochitoninae, 198 pp. BrillBackhuys, Leiden.

Kaas P \& RA Van Belle. 1987. Monograph of living chitons (Mollusca: Polyplacophora). Vol. 3. Suborder Ischnochitonidae. Chaetopleurinae and Ischnochitoninae. Addition to vols. 1 and 2, 302 pp. Brill-Backhuys, Leiden.

Kaas P \& RA Van Belle. 1990. Monograph of living chitons (Mollusca: Polyplacophora). Vol. 4. Suborder Ischnochitonina: Ischnochitonidae: Ischnochitoninae. (cont.). Addition to vols. 1, 2 and 3, 315 pp. Brill-Backhuys, Leiden.

Little C \& JA Kitching. 1996. The biology of rocky shores, 240 pp. Oxford University Press, Oxford. 
Lowell RB. 1984. Desiccation of intertidal limpets: effects of shell size, fit to substratum, and shape. Journal of Experimental Marine Biology and Ecology 77: 197-207.

Lyons WG. 1988. A review of Caribbean Acanthochitonidae (Mollusca: Polyplacophora) with description of six new species of Acanthochitona Gray, 1821. American Malacological Bulletin 6: 79-114.

Lyons WG \& F Moretzsohn. 2009. Polyplacophora (Mollusca) of the Gulf of Mexico. In: Felder DL \& DK Camp (eds). Gulf of Mexico: origins, waters, and biota. Vol. 1: Biodiversity, pp. 569-578. Texas A\&M University Press, Corpus Christi.

Martín-del Pozzo AL. 1997. Geología. En: González-Soriano E, R Dirzo \& R Vogt (eds). Historia natural de los Tuxtlas, pp. 25-31. Comisión Nacional para el Conocimiento y Uso de la Biodiversidad, México.

Moreno-Casasola P. 2004. Las playas y las dunas del Golfo de México. Una visión de la situación actual. En: Caso M, I Pisanty \& E Ezcurra (eds). Diagnóstico ambiental del Golfo de México, pp. 491-520. Instituto Nacional de Ecología, México.

Otaíza RD \& B Santelices. 1985. Vertical distribution of chitons (Mollusca: Polyplacophora) in the rocky intertidal zone of central Chile. Journal of Experimental Marine Biology and Ecology 86: 229-240.

Reyes-Gómez A \& MA Salcedo-Vargas. 2002. The recent Mexican chiton (Mollusca: Polyplacophora) species. The Festivus 34: 17-27.

Reyes-Gómez A, D Ortigosa \& N Simoes. 2017. Chitons (Mollusca, Polyplacophora) from Alacranes Reef, Yucatan, Mexico. Zookeys 665: 1-36.
Schwabe E. 2008. A summary of reports of abyssal and hadal Monoplacophora and Polyplacophora (Mollusca). Zootaxa 1866: 205-222.

Schwabe E. 2010. Illustrated summary of chiton terminology (Mollusca, Polyplacophora). Spixiana 33: 171-194.

Sigwart JD, PA Green \& SB Crofts. 2015. Functional morphology in chitons (Mollusca, Polyplacophora): influences of environment and ocean acidification. Marine Biology 162: 2257-2264.

Smith D. 2013. Ecology of the New Zealand rocky shore community, 55 pp. New Zealand Marine Studies Centre, University of Otago, Otago.

Twilley RR, EJ Barron, HL Gholz, MA Harwell, RL Miller, DJ Reed, JB Rose, EH Siemann, RG Wetzel \& RJ Zimmerman. 2001. Confronting climate change in the Gulf coast region, 82 pp. Union of Concerned Scientists and Ecological Society of America, Washington.

USNOO. 1965. Oceanographic atlas of the North Atlantic Ocean. Section 1: Tides and currents, 75 pp. Publ. 700, United States Naval Oceanographic Office, Washington.

Watters T. 1991. Utilization of a simple morphospace by polyplacophorans and its evolutionary implications. Malacologia 33: 221-240.

Yáñez-Arancibia A \& JW Day. 1982. Ecological characterization of Terminos Lagoon, a tropical lagoon estuarine system in the Southern Gulf of Mexico. In: Lasserre P \& H Postma (eds). Proceedings of the International Symposium on Coastal Lagoons SCOR/ IABO/UNESCO Bordeaux, 8-14 September 1981. Oceanologica Acta 5(4, suppl.): 431-440.

Zar JH. 1999. Biostatistical analysis, 663 pp. Prentice Hall, Englewood Cliffs.

Recibido 16 de mayo de 2017 y aceptado el 14 de diciembre de 2017

Editor: Claudia Bustos D. 